\title{
Über die Verbreitung der unentbehrlichen anorganischen Nährstoffe in den Keimlingen von Phaseolus vulgaris. II.
}

\author{
Von Leopold Ritter von Portheim und Max Samec.
}

In unserer ersten Mitteilung ${ }^{1}$ ) über diesen Gegenstand haben wir über Untersuchungen berichtet, welche den Zweck hatten, die Verteilung der unentbehrlichen anorganischen Nährstoffe in Keimlingen von Phaseolus vulgaris festzustellen, wenn die Versuchspflanzen unter sonst gleichen Vegetationsbedingungen in normaler Knopscher Nährlösung oder in einer kalkfreien Lösung gezogen wurden.

Im nachfolgenden soll über Versuche referiert werden, welche eingeleitet wurden, um zu untersuchen, in welchen Mengen diese Stoffe aufgenommen werden können und wie dieselben in der Pflanze verteilt sind, wenn nur einzelne von ihnen oder bestimmte Gemische derselben den Bohnenkeimlingen dargeboten werden.

Wir beschränkten uns vorläufig darauf, diese Fragen für Kalzium und Magnesiumsalze zu prüfen, was zum Teil Nr. 7 und 9 unseres Arbeitsplanes entspricht ${ }^{2}$ ).

Die Versuchsanordnung war im großen und ganzen die glEiche wie bei unserer früheren Arbeit $\left.{ }^{3}\right)$.

Bei den einzelnen Versuchen wurden 180-310 Keimlinge von Phaseolus vulgaris per $1 \frac{1}{2}-3$ Liter Nährlösung verwendet.

Die als Kulturgefäße dienenden Porzellanschalen wurden diesmal alle mit Paraffin ausgegossen.

Zur Befestigung des Organtins benutzten wir statt des Bindfadens S-förmig gebogene Nickeldrahtstifte, deren Verwendung sich schon bei einer anderen Gelegenheit als sehr praktisch' erwies ${ }^{4}$ ).

Die Versuche gelangten in der Zeit von Oktober 1907 bis Februar 1908 zur Aufstellung.

Die Kulturen einer Versuchsreihe befanden sich unter den gleichen Licht-, Temperatur- $\left(25^{\circ} \mathrm{C}\right.$ maximal $)$ und Feuchtigkeitsverhältnissen, verschieden waren nur die Kulturflüssigkeiten.

1) Pọtheim, v. L. u. Samec, M., Über die Verbreitung der unentbehrlichen anorganischen Nährstoffe in den Keimlingen von Phaseolus vulgaris I. Flora oder Allg. bot. Zeitung 1905, Bd. 94, H. 2, pag. 263.

2) Portheim, v. L. u. Samec, M., l. c., pag. 266.

3) Portheim, v. L. u. Samec, M., l. c., pag. $266 \mathrm{ff}$.

4) Portheim, v. L. u. Samec, M., Orientierende Untersuchungen über die Atmung gesunder und infolge von Kalkmangel erkrankter Keimlinge von Phaseolus vulgaris. Wiesner-Festschrift, Wien 1908, pag. 114. 
Als solche wurden verwendet:

Destilliertes Wasser,

$\frac{1}{50}$ Normallösung von $\mathrm{Mg}\left(\mathrm{NO}_{3}\right)_{2}$,

\begin{tabular}{|c|c|c|c|}
\hline$\frac{1}{100}$ & $"$ & $"$ & $"$ \\
\hline$\frac{1}{50}$ & $"$ & $"$ & $\mathrm{Ca}\left(\mathrm{NO}_{3}\right)_{2}$ \\
\hline$\frac{1}{100}$ & $"$ & $"$ & 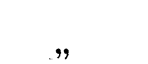 \\
\hline
\end{tabular}

Ein Gemisch von gleichen Teilen von $\frac{1}{50}$ Norm. $\operatorname{Mg}\left(\mathrm{NO}_{3}\right)_{2}$ und $\frac{1}{50}$ Norm. $\mathrm{Ca}\left(\mathrm{NO}_{3}\right)_{2}$,

$$
\begin{aligned}
& \text { " " " } \quad \text { zwei Teilen von } \frac{1}{100} \ldots, \quad \text {, , einem } \\
& \text { Teil } \frac{1}{100} \text { Norm. } \mathrm{Ca}\left(\mathrm{NO}_{3}\right)_{2} \text {, } \\
& \text { " " } \quad \text {, einem Teil von } \frac{1}{100} \quad \text { " , \# zwei } \\
& \text { Teilen } \frac{1}{100} \text { Norm. } \mathrm{Ca}\left(\mathrm{NO}_{3}\right)_{2} \text {. }
\end{aligned}
$$

Die salpetersauren Salze von Kalzium und Magnesium kamen als Lösungen gleicher Normalität in Verwendung um wesentliche osmo'tische Druckdifferenzen zu vermeiden. - Der Grundgedanke bei unseren Versuchen entspricht der von Benecke ${ }^{1}$ ) ausgesprochenen Ansicht, daß es sich zunächst darum handeln würde, die Wirkung möglichst vieler Salze, einzeln oder kombiniert und in mannigfach variierter Konzentration und Reaktion zu untersuchen.

Die Versuche wurden zu verschiedenen, aus der beigeschlossenen Tabelle ersichtlichen Zeiten abgebrochen, entweder einen Tag nach der Erkrankung der in Magnesiumnitrat allein kultivierten Keimlinge, oder in dem Stadium, in dem die Bohnen der Kulturen mit kombinierten Lösungen hinter denen in $\mathrm{Ca}\left(\mathrm{NO}_{3}\right)_{2}$-Lösungen allein oder in der Mischung von 2 Teilen Kalziumnitrat und 1 Teil Magnesiumnitrat zurückblieben.

Bezüglich der chemischen Untersuchung verweisen wir auch auf unsere erste Mitteilung ${ }^{2}$ ).

1) Benecke, W., Einige neuere Untersuchungen-über den Einfluß von Mineralsalzen auf Organismen. Botanische Zeitung 1904, Bd. LXII, 2. Abt., pag. $113,118$.

2) Portheim, v. L. u. Samec, M., l. c., pag. $268 \mathrm{ff}$. 
Die Bestimmung der Trockensubstanz erfolgte in der Weise, daß die Keimlinge zuerst durch zwei Tage auf dem Wasserbade erhitzt wurden, dann kamen sie in den Trockenschrank, wo sie bei $110^{\circ}$ verblieben, bis in den Zehnteln des Gewichtes Konstanz eintrat.

Der Kalk wurde als Oxalat in essigsaurer Lösung, Magnesia als $\mathrm{MgNH}_{4} \mathrm{PO}_{4}$ und die Phosphorsäure als Ammoniummolybdänphosphat, welches bei $160^{\circ}$ bis zur Gewichtskonstanz erhitzt wurde, bestimmt.

Die Resultate der Analysen sind in der Tabelle auf pag. 268 u. 269 zusammengestellt. Es wird der Gehalt von 100 Teilen Asche an CaO. $\mathrm{MgO}, \mathrm{K}_{2} \mathrm{O}, \mathrm{P}_{2} \mathrm{O}_{5}, \mathrm{SO}_{3}$, $\mathrm{Cl}$ und $\mathrm{Fe}_{2} \mathrm{O}_{3}$ angegeben und berechnet, wieviel von diesen Stoffen in 250 Keimlingen enthalten war. Außer diesen Daten enthält die Tabelle noch folgende Angaben:

Nummer der Versuchsreihe, Art der Nährlösung, Dauer des Versuches, Termin der Erkrankung der in destilliertem Wasser oder Magnesiumnitrat allein gezogenen Keimlinge, Länge der Pflanzen, Gewicht der Trockensubstanz und der Asche, Aschengehalt der Keimlinge in Prozenten der Trockensubstanz.

Auch 250 angekeimte Samen des zu unseren Versuchen verwendeten Bohnenmaterials wurden einer chemischen Untersuchung unterworfen, die diesbezüglichen Ergebnisse sind in der Tabelle bei der zweiten Versuchsreihe angeführt. Wir beschränkten uns auf diese Untersuchung, da das Material für alle Versuche stets das gleiche blieb und außerdem die Resultate der. Aschenanalysen der in destilliertem Wasser gezogenen Bohnen zum Vergleich herangezogen werden konnten.

Was nun die Entwicklung der Bohnen in den verschiedenen Kulturen betrifft, so gelangten wir zu folgenden Ergebnissen:

Die in destilliertem Wasser oder in einer Lösung von Magnesiumnitrat gezogenen Keimlinge von Phaseolus vulgaris erkrankten (Vers. I nach 5 Tagen, 15 und $11 \mathrm{~cm}$ lang, Vers. II nach 7 Tagen, 7 und 4,5 cm lang, Vers. III nach 6 Tagen, $11-12 \mathrm{~cm}$ lang, Vers. IV nach 10 Tagen $12-13 \mathrm{~cm}$ lang, Vers. $V$ nach 11 Tagen $3-9 \mathrm{~cm}$ lang).

In den Magnesiakulturen waren die Wurzeln ganz kurz, stark gebräunt, viele faulten. Am Hypokotyl trat die Erkrankung unter der Nutation oder unterhalb der Kotyledonen auf. Die erkrankte Stelle schnürte sich meistens ein und war gebräunt oder faulend. Mitunter traten unregelmäßige braune Flecke an dem Hypokotyl und wo das Epikotyl bereits entwickelt war, auch an diesem auf. In den verdünnten Lösungen erfolgte die Erkrankung etwas später als in den konzentrierten. 
Über die Verwendung der unentbehrlichen anorganischen Nährstoffe usw. 263

Die Keimlinge der Kulturen in destilliertem Wasser wurden etwas länger als die der $\mathrm{Mg}\left(\mathrm{NO}_{3}\right)_{2}$-Kulturen, die Wurzeln stellten das Wachstum nicht so frühzeitig ein, und waren nicht so stark gebräunt.

Die Bohnen dieser beiden Kulturen blieben gegenüber denen in den anderen Lösungen im Wachstum stark zurück.

Die Keimpflanzen, denen bloß Kalziumnitrat zur Verfügung stand, zeigten eine normale Entwicklung, eine Erscheinung, auf die bereits Böhm ${ }^{1}$ ) und Liebenberg ${ }^{2}$ ) hingewiesen haben. Diese Keimlinge waren stets bedeutend länger, als die ohne Kalk kultivierten (Vers. I 28 , cm, Vers. II $19-20 \mathrm{~cm}$, Vers. III $31-32 \mathrm{~cm}$, Vers. IV $30 \mathrm{~cm}$, Vers. V 23 und $30-43 \mathrm{~cm}$ ).

Wurde den Pflanzen außer salpetersaurem Kalk auch salpetersaure Magnesia zugeführt, so wurde das Wachstum hierdurch in verschiedener Weise beeinflußt, je nach dem Verhältnis, in welchem die Mischung Ca und $\mathrm{Mg}$ enthielt und je nach der Dauer der Kultur. War $\frac{\mathrm{CaO}}{\mathrm{MgO}}=\frac{56,10}{40,36}=1,39$, so waren die Keimlinge bei kurzer Versuchsdauer ( 6 bis 7 Tage) kürzer, bei länger währender Kultur (11 Tage) auch kürzer oder gleich lang, wie die in Kalziumnitrat allein gezogenen Bohnen.

Betrug der Kalkfaktor 2,78, so waren die Versuchspflanzen länger als die $\mathrm{Ca}\left(\mathrm{NO}_{3}\right)_{2}$-Keimlinge, es war gleichgültig ob sie kürzere (7 Tage) oder längere (11 Tage) Zeit hindurch kultiviert wurden.

War das Verhältnis von $\mathrm{CaO} \mathrm{zu} \mathrm{MgO}=0,69$, so waren einige Keimlinge im Wachstum ganz zurückgeblieben, andere hatten sich schön entwickelt, waren aber nach 7 und 12 Tagen kürzer als die Kalkpflanzen; war $\frac{\mathrm{CaO}}{\mathrm{MgO}}=1,39$, so waren die Keimlinge zunächst besser gewachsen, als die in der Kalklösung, später blieben sie aber zürück. An manchen Wurzeln waren die Spitzen weich und einige Exemplare faulten an den obérirdischen Organen.

Nur wenn der Kalkfaktor $=2,78$ war, standen die Pflanzen der Kalzium-Magnesium-Mischungen am besten.

1) Böhm, Josef, Über den vegetabilischen Nährwert der Kalksalze. Sitzungsberichte der k. Akademie der Wissenschaften zu Wien, 1875, I. Abt., LXXI, Aprilheft, Sep. pag. 10.

2) Liebenberg, Ritter, v. A., Untersuchungen über die Rolle des Kalkes bei der Keimung von Samen. Sitzungsberichte der k. Akademie der Wissenschaften zu Wien, I. Abt., 1881, LXXXIV, III. Heft, pag. 405, 414. 
Es stimmt dies mit den Resultaten von Daikuhara ${ }^{1}$ ) überein, welcher Phaseolus ${ }^{2}$ ) in Quarzsand kultivierte, dem alle notwendigen Nährstoffe zugesetzt wurden, bloß das Verhältnis von Kalk zu Magnesia wurde von $\frac{3}{1}$ bis $\frac{0,33}{1}$ variiert. Nur wenn $\frac{\mathrm{CaO}}{\mathrm{MgO}} \frac{2}{1}$ betrug, so wurden die höchsten Pflanzen mit den größten Blättern erzielt.

Während den Pflanzen Daikuharas alle Nährstoffe zur Verfügung standen, wurden unsere Keimlinge nur in Kalzium- und Magnesiumnitrat kultiviert, trotzdem gelangten wir zu sehr ähnlichen Resultaten ${ }^{2}$ ); dies spricht dafür, daß bei der Erkrankung der Phaseolus-Keimlinge

Samen, und Kulturen in destilliertem Wasser:

\begin{tabular}{|c|c|c|c|c|c|c|c|c|}
\hline Kultur & $\begin{array}{c}\text { Ver- } \\
\text { suchs- } \\
\text { reihe }\end{array}$ & $\begin{array}{c}\text { Dauer } \\
\text { des } \\
\text { Versuches }\end{array}$ & $\begin{array}{c}\text { Asche } \\
\mathrm{g}\end{array}$ & $\begin{array}{l}\text { In } 100 \\
\text { Asche } \\
\text { enth } \\
\mathrm{CaO}\end{array}$ & $\begin{array}{l}\text { Teilen } \\
\text { waren } \\
\text { alten } \\
\mathrm{MgO}\end{array}$ & $\begin{array}{c}\text { Verhältnis } \\
\text { von } \\
\mathrm{CaO}: \mathbf{M g O}\end{array}$ & $\begin{array}{l}1 \text { Teil } \\
\text { CaO } \\
\text { entfie }\end{array}$ & $\begin{array}{l}1 \text { Teil } \\
\text { MgO } \\
\text { af Teile } \\
\text { che }\end{array}$ \\
\hline $\begin{array}{c}\text { Samen } \\
\text { destill. Wasser } \\
\qquad,\end{array}$ & $\begin{array}{l}\text { II. } \\
\text { II. }\end{array}$ & $\begin{array}{l}6 \text { Tage } \\
8 \bar{y}\end{array}$ & $\begin{array}{l}2,435 \\
2,208 \\
2,020\end{array}$ & $\begin{array}{l}2,44 \\
2,98 \\
2,72\end{array}$ & $\begin{array}{l}7,40 \\
7,64 \\
8,22\end{array}$ & $\begin{array}{l}0,33: 1 \\
0,39: 1 \\
0,33: 1\end{array}$ & $\begin{array}{l}41,27 \\
33,45 \\
36,73\end{array}$ & $\begin{array}{l}13,53 \\
13,07 \\
12,17\end{array}$ \\
\hline
\end{tabular}

Kulturen in Lösungen von $\mathrm{Mg}\left(\mathrm{NO}_{3}\right)_{2}$.

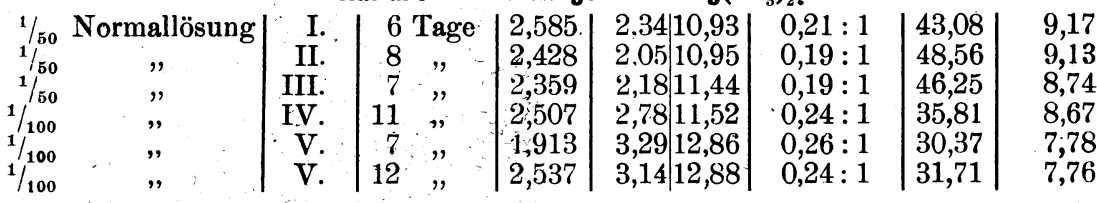

Kulturen in Lösungen von $\mathrm{Ca}\left(\mathrm{NO}_{3}\right)_{2}$

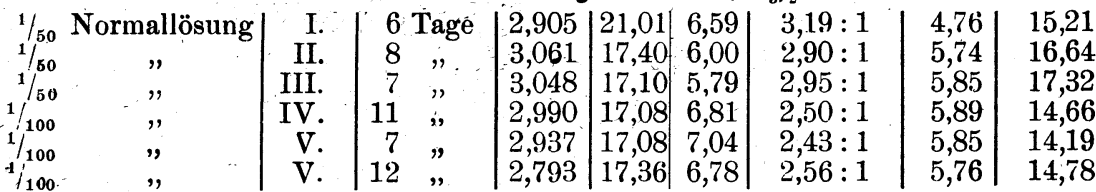

Gleiche Teile von $\mathrm{Mg}\left(\mathrm{NO}_{3}\right)_{2}$ und $\mathrm{Ca}\left(\mathrm{NO}_{3}\right)_{2}$-Lösungen

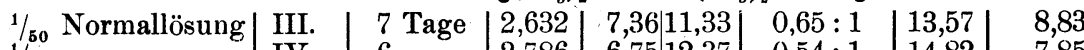

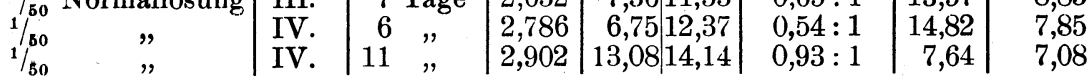

Zwei Teile einer $\mathrm{Ca}\left(\mathrm{NO}_{3}\right)_{2}$-Lösung und ein Teil einer $\mathrm{Mg}\left(\mathrm{NO}_{3}\right)_{2}$-Lösung

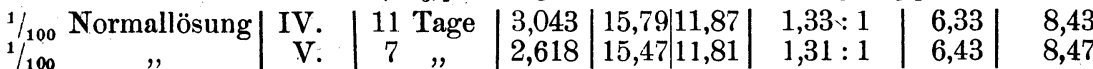

Zwei Teile einer $\mathrm{Mg}\left(\mathrm{NO}_{3}\right)_{2}$-Lösung und ein Teil einer $\mathrm{Ca}\left(\mathrm{NO}_{3}\right)_{2}$-Lösung $1 / 100$ Normallösung $\mid$\begin{tabular}{l|l|l|l|l|l|l|}
7 & Tage & 2,721 & $8,60 \mid 15,56$ & $0,55: 1$ & $11,63 \mid$ & 6,43
\end{tabular}

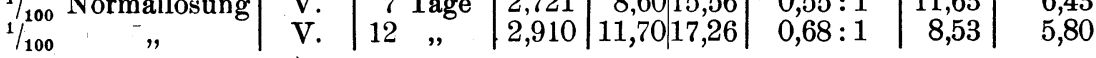

1) Daikuhara, G., On the Influence of different Ratios between Lime and Magnesia upon the Development of Phaseolus. The Bulletin of the College of Agriculture. Tokyo Imperial University, Vol. V, 1902,03, pag. 501.

2) Leider ist aus der Abhandlung nicht zu ersehen, welche Phaseolus-Spezies Daikuhara untersuchte. 
in destilliertem Wasser, in Lösungen von $\mathrm{Mg}\left(\mathrm{NO}_{3}\right)_{2}$ und in kalkfreien Nährlösungen das Verhältnis von $\mathrm{Ca}: \mathrm{Mg}$ wenn auch nicht die einzige, so doch eine der Ursachen ist.

Im. nachfolgenden sollen nun die bei der Untersuchung der Asche der diversen Versuchspflanzen erzielten Resultate besprochen werden.

Bei Durchsicht der Zusammenfassung auf Seite 264 fällt es auf, daß bei Versuchen mit Magnesiumnitrat allein, welche nur kurze Zeit währten, größere Mengen von Kalk im Verhältnis zu Magnesia. nachzuweisen waren als bei länger dauernden Kulturen. Da den Keimlingen Kalk nicht zur Verfügung stand, geht daraus die stetige Aufnahme von $\mathrm{Mg}$ während der Kultur hervor; dieselbe scheint im Anfange intensiver zu sein als später. Bei stärkerer Konzentration der Magnesiumlösung ( $1 / 50$ Normallösung) stieg der Magnesiumgehalt zirka auf das Fünffache, bei geringer Konzentration ( $1 / 100$ Normallösung) bloß auf das Vierfache der in den Samen vorhandenen Kalziummenge.

Die Aufnahme des $\mathrm{Ca}$ aus den $\mathrm{Ca}\left(\mathrm{NO}_{3}\right)_{2}$-Lösungen wurde auch durch die Konzentration der Lösung beeinflußt. Bei den Kulturen in $1 / 50$ Normallösung war zirka dreimal soviel $\mathrm{Ca}$ als $\mathrm{Mg}$, bei den Kulturen in $1 / 100$ Normallösung ca. 2,5 mal soviel $\mathrm{Ca}$ als $\mathrm{Mg}$ festzustellen. Die Keimlinge dieser Versuche blieben gesund.

Wurde der Kalziumnitratlösung, in der die Keimlinge gezogen wurden, auch Magnesiumnitrat zugesetzt, so hatte dies stets eine Herabminderung der Kalziumaufnahme zur Folge und zwar umso stärker, je mehr $\mathrm{Mg}\left(\mathrm{NO}_{3}\right)_{2}$ zugeführt wurde. Während des Versuches nahm die Kalkmenge zu, erreichte aber niemals die bei Ernährung mit salpetersaurem Kalk allein gefundenen Quantitäten. Fiel das Verhältnis von $\frac{\mathrm{CaO}}{\mathrm{MgO}}$ unter 1 , so entwickelten sich die Keimlinge schlecht oder es warén an ihnen Krankheitserscheinungen $\mathrm{zu}$ beobachten.1)

Eine Bestätigung des eben Mitgeteilten ergibt auch eine Vergleichung der Zahlen, welche angeben, auf wieviel Teile der Asche ein Teil Kalziumoxyd oder Magnesiumoxyd entfällt. In den im destillierten Wasser gezogenen Keimlingen und in den Samen war zirka dreimal mehr $\mathrm{MgO}$ als $\mathrm{CaO}$ nachweisbar. Wurden solche Samen ohne Kalk-

1) Die Veränderung des Verhältnisses von $\mathrm{CaO}: \mathrm{MgO}$ in Pflanzen, welche in Substraten gezogen wurden, deren Kalkgehalt variiert wurde, untersuchten Loew, O. u. Aso, K. (The Bulletin of the College of Agriculture, Tokyo, Vol. VI, Nr. 4, pag. 335) u. Takeuchi, T. (The Bulletin of the College of Agriculture, Tokyo, Vol. VII, 1906/08, pag. 579. 
zufuhr oder in destilliertem Wasser kultiviert, so erkranken die Keimlinge.

Nach Liebenberg ${ }^{1}$ ) ist Kalk zur normalen Keimung von Brassica napus oleifera, Sinapis alba, Papaver somniferum und Carum carvi nicht notwendig. Vergleicht man nun in der folgenden, den W olf f schen ${ }^{2}$ ) Tabellen entnommenen Zusammenstellung die in 100 Teilen Asche enthaltenen Kalk- und Magnesiamengen, so findet man, daß die Samen resp. Früchte mehr Kalzium als Magnesium enthalten. Hingegen ist das Verhältnis dieser beiden Stoffe ein umgekehrtes bei denjenigen Pflanzen, deren Keimlinge nach den Befunden Liebenbergs Kalk bedürfen, wenn die Reservestoffe verbraucht werden und die Pflanzen nicht erkranken sollen. Unter den letzteren befindet sich auch Phaseolus vulgaris, unsere Versuchspflanze.

Mittlere prozentische Zusammensetzung der Asche der Samen und Früchte der von Liebenberg auf ihr Kalkbedürfnis bei der Keimung geprüften Pflanzen, nach Wolff ${ }^{3}$ ):

Keimlinge, welche bei Kalkmangel nicht erkranken:

\begin{tabular}{|c|c|c|c|c|c|}
\hline \multirow[b]{2}{*}{ Brassica napus oleifera } & $\mathrm{CaO}$ & $\begin{array}{c}\mathrm{MgO} \\
\text { In } 100\end{array}$ & $\begin{array}{l}\mathrm{K}_{2} \mathrm{O} \\
\text { en Asche }\end{array}$ & $\mathrm{P}_{2} \mathrm{O}_{5}$ & \multirow[t]{2}{*}{ Verhältnis $\mathrm{CaO}: \mathrm{MgO}$} \\
\hline & & & & & \\
\hline . & 14,1 & 11,80 & 24 & 42,3 & $1,20: 1$ \\
\hline $\mathrm{Car}$ & 18 & 8,27 & 26 & 24, & $2,18: 1$ \\
\hline Papaver & 35 & 9,4 & & 31 , & $3,73: 1$ \\
\hline Sinapis alba (weißer Senf) ${ }^{4}$ ) & 20,19 & 8,58 & 17,90 & 41,19 & $2,35: 1$ \\
\hline
\end{tabular}

Keimlinge, welche bei Kalkmangel erkranken:

\begin{tabular}{|c|c|c|c|c|c|}
\hline & $\mathrm{CaO}$ & $\begin{array}{r}\mathrm{MgO} \\
\text { In } 100 \mathrm{~T}\end{array}$ & $\underset{\text { ilen Asche }}{\mathrm{K}_{2} \mathrm{O}}$ & $\mathrm{P}_{2} \mathrm{O}_{5}$ & Verhältnis von $\mathrm{CaO}: \mathrm{MgO}$ \\
\hline Cannabis sativa (Hanf) & 23,64 & 5,70 & 20,28 & 36,46 & $4,15: 1$ \\
\hline $\begin{array}{l}\text { Linum usitatissimum (Lein). } \\
\text { Phaseolus vulgaris (Garten- }\end{array}$ & 8,10 & 14,29 & 30,63 & 41,50 & $0,57: 1$ \\
\hline bohne). & 6,38 & 7,62 & 44,01 & 35,52 & $0,84: 1$ \\
\hline $\begin{array}{l}\text { Pisum sativum (Erbse) } \\
\text { Polygonum fagopyrum (Buch- }\end{array}$ & 4,81 & 7,99 & 43,10 & 35,90 & $0,60: 1$ \\
\hline weizen) & 4,4 & 12,42 & 23,07 & 48,67 & $0,36: 1$ \\
\hline Soja hispida (Sojabohne & 5,32 & 8,92 & 44,56 & 36,89 & $0,60: 1$ \\
\hline Vicia sativa (Futterwicke) & 8,03 & 8 & 30 & & $0,90: 1$ \\
\hline Zea Mays (Mais) & 2,17 & 15,52 & 29,78 & 45,61 & $0,14: 1$ \\
\hline
\end{tabular}

Von diesen bei Kalkmangel zugrunde gehenden Pflanzen enthält nur Cannabis sativa mehr Kalk als Magnesia in den Samen. Es soll die Aufgabe weiterer Untersuchungen sein, bei dieser Pflanze die Ur-

1) Liebenberg, R. v. A., l. c., pag. 434 .

2) Wolff, Emil, Aschenanalysen von landwirtschaftlichen Produkten, Fabrik abfällen und wildwachsenden Pflanzen. I. u. II. Teil, 1871 u. 1880 . Berlin.

3) Wolff, E., Aschenanalysen, II. Teil 1880, pag. 123.

4) Wolff, E., Aschenanalysen, I. Teil, 1871, pag. 106. 
sache der Erkrankung bei Kultur in kalkfreien Medien festzustellen. Es sei darauf hingewiesen, daß der eine ${ }^{1}$ ) von uns zeigen konnte, daß Keimlinge von Carum Carvi, welche nach Liebenberg, in kalkfreier Nährlösung gezogen, gesund bleiben, unter Umständen in Nährlösungen, denen der Kalk fehlt, erkranken können.

Ebenso wie die in destilliertem Wasser gezogenen Keimlinge von Phaseolus vulgaris erkrankten auch stets alle bloß mit Magnesiumnitrat ernährten Bohnen. Es wurde bereits früher erwähnt, daß die letzteren, was die Wurzeln und die oberirdischen Organe betrifft, die Länge der ersteren nicht erreichten.

Die absoluten von den Phaseolus-Keimlingen aus den einzelnen Kulturflüssigkeiten aufgenommenen Stoffe berechneten wir aus den für 250 Keimlinge nachgewiesenen $\mathrm{CaO}$ und $\mathrm{MgO}$-Mengen (siehe Tabelle pag. 268 u. 269).

Zur Berechnung des Durchschnittswertes des ursprünglichen Kalkund Magnesiagehaltes von 250 Samen wurden die für Samen (Vers. II), für Keimlinge in destilliertem Wasser (Vers. I, II) und für Keimlinge in Lösungen von salpetersaurem Magnesium resp. von salpetersaurem Kalzium (Vers. I, II, III, IV und V) ermittelten Werte herangezogen.

Die Zahlen in der folgenden Zusammenstellung zeigen an, um wie viel mehr Kalk und Magnesia in den Keimlingen, welche in $\mathrm{Ca}\left(\mathrm{NO}_{3}\right)_{2}$ oder $\mathrm{Mg}\left(\mathrm{NO}_{3}\right)_{2}$-Lösungen, oder in Gemischen dieser beiden Nährstoffe gezogen worden waren, enthalten war als ursprünglich in den Samen.

1) Portheim, R. v. L., Über die Notwendigkeit des Kalkes für Keimlinge, insbesondere bei höherer Temperatur. Sitzungsberichte der k. Akádemie der Wissenschaften in Wien 1901, CX, I. Abt.; April, Sep. pag. 21. 


\begin{tabular}{|c|c|c|c|c|c|c|}
\hline $\begin{array}{l}\text { Ver- } \\
\text { suchs- } \\
\text { reihe }\end{array}$ & Kulturflüssigkeit & $\begin{array}{c}\text { Dauer } \\
\text { des } \\
\text { Ver- } \\
\text { suches }\end{array}$ & $\begin{array}{c}\text { Er- } \\
\text { krankung } \\
\text { nach } \\
\text { Tagen }\end{array}$ & $\begin{array}{c}\begin{array}{c}\text { Länge } \\
\text { der } \\
\text { Keimlinge }\end{array} \\
\mathbf{c m}\end{array}$ & $\begin{array}{c}\text { Gewicht der } \\
\text { Trockensub- } \\
\text { stanz von 250 } \\
\text { Keimlingen } \\
\mathrm{g}\end{array}$ & \begin{tabular}{|c} 
Gewicht \\
der Asche \\
von 250 \\
Keim- \\
lingen \\
g \\
\end{tabular} \\
\hline I. & Destilliertes Wasser. . . . . & 6 & 5 & 15 & 34,79 & 2,208 \\
\hline I. & $1 / 50$ Normall. $\mathrm{Mg}\left(\mathrm{NO}_{3}\right)_{2}$. . . & 6 & 5 & 11 & 43,57 & 2,585 \\
\hline I. & ${ }^{1 / 50}$ Normall. $\mathrm{Ca}\left(\mathrm{NO}_{3}\right)_{2} \cdot \ldots$ & 6 & - & 28 & 36,91 & 2,905 \\
\hline II. & Samen . . . . . . . . . . & - & - & - & 62,90 & 2,435 \\
\hline II. & Destilliertes Wasser. . . . . & 8 & 7 & 7 & 39,03 & 2,020 \\
\hline II. & $1 / 50$ Normall. $\mathrm{Mg}\left(\mathrm{NO}_{3}\right)_{2}$. . . & 8 & 7 & 4,5 & 51,16 & 2,428 \\
\hline II. & ${ }^{1}{ }_{150}$ Normall. $\mathrm{Ca}\left(\mathrm{NO}_{3}\right)_{2} \ldots \ldots$ & 8 & - & $19-20$ & 38,46 & 3,061 \\
\hline III. & $1 / 50$ Normall. $\mathrm{Mg}\left(\mathrm{NO}_{3}\right)_{2}$. . . & 7 & 6 & $11-12$ & 44,2 & 2,359 \\
\hline III. & $\begin{array}{l}1 \text { Teil } 1 / 50 \text { Normall. } \mathrm{Mg}\left(\mathrm{NO}_{3}\right)_{2}+ \\
1 \text { Teil } 1 / 50 \text { Normall. } \mathrm{Ca}\left(\mathrm{NO}_{3}\right)_{2}\end{array}$ & 7 & - & 30 & 40,3 & 2,632 \\
\hline III. & $1 / 50$ Normall. $\mathrm{Ca}\left(\mathrm{NO}_{3}\right)_{2} \ldots$ & 7 & - & $31-32$ & 39,13 & 3,048 \\
\hline IV. & 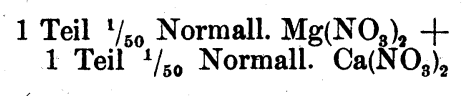 & 6 & - & 14 & 51,6 & 2,786 \\
\hline IV. & $\begin{array}{r}1 \text { Teil } 1 / 50 \text { Normall. } \mathrm{Mg}\left(\mathrm{NO}_{3}\right)_{2}+ \\
1 \text { Teil } 1 / 50 \text { Normall. } \mathrm{Ca}\left(\mathrm{NO}_{3}\right)_{2}\end{array}$ & 11 & - & 30 & 44,6 & 2,902 \\
\hline IV. & $1 / 400$ Normall. $\mathrm{Mg}\left(\mathrm{N}_{3}\right) \mathrm{O}_{2}$. . . & 11 & 10 & $12-13$ & 50,7 & 2,507 \\
\hline IV. & $\begin{array}{l}2 \text { Teile }{ }^{\mathrm{T}} / 100 \text { Normall. } \mathrm{Ca}\left(\mathrm{NO}_{\mathrm{B}}\right)_{2}+ \\
1 \text { Teil }{ }_{1 / 100} \text { Normall. } \mathrm{Mg}\left(\mathrm{NO}_{3}\right)_{2}\end{array}$ & 11 & - & 33 & 46,5 & 3,043 \\
\hline IV. & ${ }^{1} /{ }_{100}$ Normall. $\mathrm{Ca}\left(\mathrm{NO}_{3}\right)_{2} \cdot$ & 11 & - & 30 & 45,7 & 2,990 \\
\hline V. & $1 / 100$ Normall. $\mathrm{Ca}\left(\mathrm{NO}_{3}\right)_{2} \ldots$. & 7 & - & 23 & 48,9 & 2,937 \\
\hline V. & $1 / 100$ Normall. $\mathrm{Mg}\left(\mathrm{NO}_{3}\right)_{2} \ldots \ldots$ & 7 & - & $5-9$ & 56,8 & 1,913 \\
\hline V. & $1 / 100$ Normall. $\mathrm{Ca}\left(\mathrm{NO}_{3}\right)_{2} \cdot \ldots$ & 12 & - & $30-38-43$ & 38,0 & 2,793 \\
\hline V. & $1 / 100$ Normall. $\mathrm{Mg}\left(\mathrm{NO}_{3}\right)_{2}$ & 12 & 11 & $3-4-9$ & 54,7 & 2,537 \\
\hline V. & $\begin{array}{l}2 \text { Teile } 1 / 100 \text { Normall. } \mathrm{Ca}\left(\mathrm{NO}_{3}\right)_{2}+ \\
1 \text { Teil }^{1} / 100 \text { Normall. } \mathrm{Mg}\left(\mathrm{NO}_{3}\right)_{2}\end{array}$ & 7 & - & 24 & 47,7 & 2,618 \\
\hline V. & $\begin{array}{l}2 \text { Teile } 1 /{ }_{100} \text { Normall. } \mathrm{Mg}\left(\mathrm{NO}_{3}\right)_{2}+ \\
1 \text { Teil }{ }_{1 / 100} \text { Normall. } \mathrm{Ca}\left(\mathrm{NO}_{3}\right)_{2}\end{array}$ & 7 & - & 22 & 48,0 & 2,721 \\
\hline V. & $\begin{array}{r}2 \text { Teile }^{1 /} /{ }_{100} \text { Normall. } \mathrm{Mg}\left(\mathrm{NO}_{3}\right)_{2}+ \\
1 \text { Teil } 1 / 100 \text { Normall. } \mathrm{Ca}\left(\mathrm{NO}_{3}\right)_{2}\end{array}$ & 12 & - & $31-37$ & 43,3 & 2,910 \\
\hline
\end{tabular}


Über die Verbreitung der unenthehrlichen anorganischen Nährstoffe usw. 269

\begin{tabular}{|c|c|c|c|c|c|c|c|c|c|c|c|}
\hline \multirow{2}{*}{$\begin{array}{l}\text { Asche in } \\
\text { Prozenten } \\
\text { der } \\
\text { Trocken- } \\
\text { substanz }\end{array}$} & \multicolumn{6}{|c|}{$\begin{array}{c}\text { In } 100 \text { Teilen Asche waren enthalten } \\
\text { Teile: }\end{array}$} & \multicolumn{4}{|c|}{$\begin{array}{l}\text { Die Asche von } 250 \\
\text { Keimlingen enthält } \\
\mathrm{g}\end{array}$} & \multirow[t]{2}{*}{ Anmerkungen } \\
\hline & $\mathrm{CaO}$ & \begin{tabular}{|l|l|}
$\mathrm{MgO}$ & $\mathrm{K}_{2} \mathrm{O}$ \\
\end{tabular} & $\mid \mathrm{P}_{2} \mathrm{O}_{5}$ & $\mathrm{SO}_{3}$ & $\mathrm{Cl}$ & $\mathrm{Fe}_{2} \mathrm{O}_{3}$ & $\mathrm{CaO}$ & $\mid \mathrm{MgO}$ & $\mid \mathrm{K}_{2} \mathrm{O}$ & $\mathrm{P}_{2} \mathrm{O}_{5}$ & \\
\hline 6,35 & 2,98 & $7,64 \mid 48,93$ & 29,97 & 10,21 & 0,36 & 0,25 & 0,066 & 0,169 & 1,080 & $0,0,662$ & \\
\hline 5,94 & 2,34 & $10,9349,16$ & 27,93 & 8,98 & 0,48 & 0,17 & 0,060 & 0,282 & 1,271 & 0,722 & \\
\hline 7,87 & 21,01 & $\begin{array}{ll}6,59 & 38,96\end{array}$ & 23,30 & 9,51 & 0,23 & 0,41 & 0,610 & 0,191 & 1,132 & 20,677 & \\
\hline 3,87 & 2,44 & $7,4050,00$ & 33,66 & 6,67 & 0,20 & 0,21 & 0,059 & 90,180 & 1,217 & 70,820 & \\
\hline 5,15 & 2,72 & $8,22 \mid 47,18$ & 331,42 & 9,89 & 0,46 & 0,14 & 0,055 & 50,166 & 0,953 & 30,635 & \\
\hline 4,75 & 2,05 & $10,95 \mid 45,99$ & 30,94 & 9,36 & 0,77 & 0,04 & $0,1) 50$ & 0,266 & 1,117 & 70,751 & \\
\hline 7,96 & 17,40 & $6,0040,75$ & 25,65 & 9,38 & 0,44 & 0,40 & 0,533 & 30,184 & 1,247 & 0,785 & \\
\hline 5,34 & 2,18 & $11,4447,87$ & 28,73 & \begin{tabular}{|l|l}
9,41 \\
\end{tabular} & 0,21 & 0,15 & 0,051 & 0,270 & 1,129 & 90,678 & \\
\hline 6,35 & 7,36 & $11,3339,80$ & 29,00 & 10,46 & 1,99 & 0,04 & 0,194 & 40,298 & 1,048 & 80,763 & \\
\hline 7,54 & 17,10 & $5,7941,20$ & 26,76 & 8,15 & 0,91 & 0,13 & 0,521 & 10,176 & 1,256 & 60,816 & \\
\hline 5,40 & 6,75 & $12,3742,27$ & 27,59 & 10,28 & 0,47 & 0,26 & 0,188 & 80,345 & 1,178 & 80,769 & $\begin{array}{l}\text { Der Versuch wurde an } \\
\text { dem Tage abgebrochen, } \\
\text { an dem die Keimlinge } \\
\text { dieser Kultur besser ent- } \\
\text { wickelt waren, als die } \\
\text { der } \mathrm{Ca}\left(\mathrm{NO}_{3}\right)_{2}-\mathrm{Kultur} \text {. }\end{array}$ \\
\hline 6,51 & 13,08 & $14,1436,74$ & 24,57 & 10,75 & 0,50 & 0,20 & 0,380 & 00,410 & 1,066 & 60,713 & \\
\hline 4,95 & 2,78 & $11,5242,13$ & 30,55 & 10,50 & 2,71 & 0,15 & 0,070 & $0,0,289$ & 1,056 & 60,766 & \\
\hline 6,55 & 15,79 & $11,8735,03$ & 24,01 & 12,65 & 0,56 & 0,09 & 0,481 & 0,361 & 1,066 & 60,731 & - \\
\hline 6,54 & 17,00 & $6,8139,21$ & 24,64 & 11,31 & 0,68 & 0,45 & 0,508 & 80,204 & 1,172 & 20,737 & \\
\hline 6,01 & 17,08 & $7,04,43,70$ & 23,39 & 9,90 & 0,61 & 0,35 & 0,502 & 20,207 & 1,284 & 40,687 & \\
\hline 3,37 & 3,29 & $12,8642,57$ & 30,43 & 8,83 & 0,83 & 0,60 & 0,063 & 30,246 & 0,814 & 40,582 & Bei dieser Versuchsreihe \\
\hline 7,35 & 17,36 & $6,7840,35$ & 24,62 & 9,82 & 0,52 & 0,53 & 0,485 & 50,189 & 1,127 & 70,680 & stand am 7. Tage die \\
\hline 4,68 & 3,14 & $12,8842,85$ & 31,36 & 8,73 & 0,19 & 0,59 & 0,080 & $0,0,327$ & 1,087 & 70,796 & 1 Teil $\left.\mathrm{Mg}\left(\mathrm{NO}_{8}\right)_{2}\right)^{3}-\mathrm{Kul}-$ \\
\hline 5,49 & 15,47 & $11,8135,57$ & 26,39 & 10,12 & 0,31 & 0,30 & 0,405 & 50,309 & 0,931 & 0,691 & $\left\{\begin{array}{l}\mathrm{Ca}\left(\mathrm{NO}_{3}\right)_{2}-\text { Kultur, wäh- } \\
\text { rend die Keimlinge der }\end{array}\right.$ \\
\hline 5,67 & 8,60 & $15,5637,45$ & 28,67 & 8,91 & 0,29 & 0,49 & 0,234 & 0,423 & 1,019 & 90,780 & $\mid \begin{array}{lll}2 & \text { Teile } & \mathrm{Mg}\left(\mathrm{NO}_{3}\right)_{2} \\
1 & \text { Teil } \mathrm{Ca}\left(\mathrm{NO}_{3}\right)_{2}-\text { Kultur } \\
\text { zurückgeblieben } & \text { waren. }\end{array}$ \\
\hline
\end{tabular}




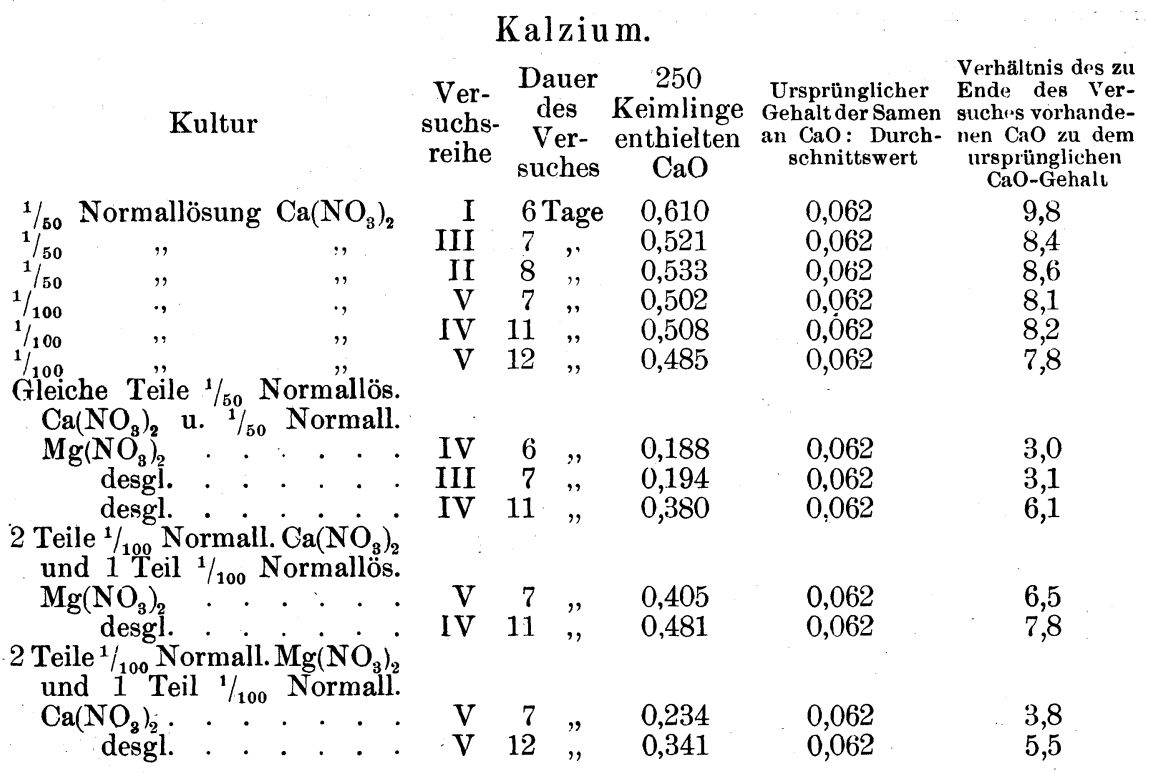

Wird den Keimlingen bloß $\mathrm{Mg}\left(\mathrm{NO}_{3}\right)_{2}$ zugeführt, so schwanken die gefundenen Werte zwischen 1,3-1,5 bei kürzerer und zwischen 1,6 und 1,8 bei längerer Versuchsdauer. Aus der verdünnten Lösung wird $\mathrm{Mg}$ langsamer aufgenommen als aus der konzentrierteren. Wird den Bohnen nun außer Magnesium auch Kalzium zur Verfügung gestellt, so hat dies eine, wenn auch nicht sehr starke Steigerung der Mg-Aufnahme zur Folge. Hier ist, ebenso wie bei der Ernährung mit Magnesiumnitrat allein, deutlich $\mathrm{zu}$ sehen, $\mathrm{da} \beta$ einer längeren Dauer des Versuches auch das Übertreten einer größeren Menge Magnesium aus der Lösung in die Pflanze entspricht. Je geringer der Kalziumgehălt der Nährlösung ist, desto mehr steigt der Magnesiumgehalt in den Keimlingen.

Die in $\mathrm{Ca}\left(\mathrm{NO}_{3}\right)_{2}$ allein kultivierten Keimlinge können das 7,8 bis 9,8 fache des ursprünglich vorhandenen Kalkes aufnehmen; wird den Bohnen außer dem salpetersauren Kalk auch salpetersaure Magnesia geboten, so hat dies eine starke Herabminderung der Kalziumaufnahme zur Folge, und zwar um so mehr, je größer der Mg-Gehalt der Lösung ist, es steigt aber der Kalkgehalt der Pflanzen während der Kultur beträchtlich. Annähernd so hohe Werte für die Ca-Aufnahme wie bei Kultur in einer Lösung von $\mathrm{Ca}\left(\mathrm{NO}_{3}\right)_{2}$ erhält man bei Keimlingen, welche in einem Gemisch von 2 Teilen $\mathrm{Ca}\left(\mathrm{NO}_{3}\right)_{2}$, und 1 Teil $\mathrm{Mg}\left(\mathrm{NO}_{3}\right)_{2}$ gezogen wurden. 
Die Mischung dieser beiden Nährstoffe hat also einerseits eine schwache Erhöhung der Magnesiumaufnahme zur Folge, andererseits wird die Aufnahme von Kalzium stark herabgedrückt.

Die ganz besonders starke Aufnahme von Kalzium durch Keimlinge, welche nur mit Kalziumnitrat ernährt wurden, ist vielleicht auf die kontinuierliche Entfernung des Kalziums aus dem Stoffwechsel durch organische Säuren zurückzuführen, während bei gleichzeitiger Zufuhr von Magnesium, welches in der Pflanze zum größten Teil in leicht löslichen Verbindungen vorkommt, die Aufnahme des Ca herabgedrückt wird. Hierfür sprechen die Untersuchungen A s os ${ }^{1}$ ), welcher fand, daß bei Kartoffeln, Buchweizen, Klee und Gerste der größte Teil der Kalksalze in Wasser unlöslich oder schwer löslich ist, während nur geringe Quantitäten leicht wasserlöslich sind. Der größte Teil der Magnesiumsalze löst sich hingegen leicht in Wasser, ein geringer Teil löst sich schwer und nur minimale Mengen, meist nur Spuren, sind unlöslich.

Die schwache Steigerung der Aufnahme des unentbehrlichen, aber für die Pflanzen mehr oder minder giftigen Magnesiums aus den Kalzium-Magnesium-Gemischen wird, wie wir vórgreifend erwähnen wollen, vielleicht durch die entgiftende Wirkung des Kalziums ermöglicht.

Unsere Untersuchungen bestätigen die bereits öfters beobachtete giftige Wirkung der Magnesiumsalze ${ }^{2}$ ).

1) Aso, K., On the different Forms of Lime in Plants. The Bulletin of the College of Agriculture, Tokyo, Imp.-Univ., 1902/03, pag. 239.

2) Benecke, W., Über die Giftwirkung verschiedener Salze auf Spirogyra und ihre Entgiftung durch Kalziumsalze. Berichte der deutschen botanischen Gesellschaft 1907, Bd. XXV, H. 6, pag. 332, 333, woselbst über die diesbezügliche Literatur referiert wird.

I, oew, O., Über die physiologischen Funktionen der Kalzium- und Magnesiumsalze im Pflanzenorganismus. Flora oder allgem. bot. Ztg. 1892, H. 3, pag. 368.

Osterhout, W. J. V., The antitoxic Action of Potassium on Magnesium. University of California Publications, Botany, Vol. II, 1906, Nr. 11, pag. 235.

Stiehr, G., Über das Verhalten der Wurzelhärchen gegen Lösungen. Inaugural-Dissertation, Kiel 1903, cit. naeh Benecke, l. c., Bot. Ztg., 1904, pag. 113.

Kearny, T. H. u. Harter, L. L., The comparative Tolerance of various Plants for the Sels common in alkali Soils. Bulletin 113. Bureau of PlantIndustry U.S. Department of Agriculture 1907. Ref, Botanisches Centralblatt 1908, Nr. 5, pag. 99. 
Aso ${ }^{2}$, Bernardini und $\left.\operatorname{Corso}^{3}\right)$, Daikuhara $\left.{ }^{4}\right)$, Furuta ${ }^{5}$, Kanomata ${ }^{6}$ ), Katayama ${ }^{7}$ ), Kumagiri ${ }^{8}$ ), Loew ${ }^{9}$ ) und Aso, Loew ${ }^{10}$ ) und Honda, Maki und Tanaka ${ }^{11}$ ), May ${ }^{12}$ ), Namikawa $\left.{ }^{13}\right)$, Sirker ${ }^{14}$ ),

2) Aso, K., The Bulletin of the College of Agriculture, Tokyo Imp.-Univ., Vol. IV, 1902, pag. 361, zit. nach Czapek, Biochemie der Pflanzen, 1905, II, pag. 850.

Ders., On the Influence of a certain Ratio between Lime and Magnesia on the Growth of the Mulberrytree. The Bulletin of the College of Agriculture, Tokyo, Imp.-Univ., Vol. V, 1902/03, pag. 495.

Ders., On the Influence of different Ratios of Iime to Magnesia on the Growth of Rice. The Bulletin of the College of Agriculture, Tokyo, Imp.-Univ., Vol. VI, 1904/05, pag. 97.

3) Bernardini, L. e Corso, G., Intorno all' influenca di vari rapporti fra calce e magnesia sullo sviluppo delle piante. Gazetta chimica italiana 1907, XXXVII, Fasc. VI, pag. 232.

4) Daikuhara, G., l. c.

5) Furuta, T., The Bulletin of the College of Agriculture, Tokyo, Imp.Univ., Vol IV, pag. 371; zit. nach Czapek, Biochemie der Pflanzen 1905, II, pag. 850.

6) Kanomata, C., On the Depression of Growth by large Doses of Lime. The Bulletin of the College of Agriculture, Tokyo, Vol. VII, 1906/08, pag. 599.

7) Katayama, T., On the Determination of the available Amounts of Lime and Magnesia in the Soil. The Bulletin of the College of Agriculture, Tokyo, Vol. VI, 1904/05, pag. 103.

8) Kumagiri, S., On the physiological Effects of an Excess of Magnesia upon Barley. The Bulletin of the College of Agriculture, Tokyo, Vol. VII, 1906/08, pag. 441.

9) Loew, O., Liming of Soils from a physiological Standpoint. The Relation of Lime ou- Magnesia to Plant Growth. U.S. Department of Agriculture, Bureau of Plant-Industry, Washington 1901, Bull. Nr. 1, pag. 9.

Loew, O. and Aso, K., On different Degrees of Availability of Plant Nutrients. The Bulletin of the College of Agriculture, Tokyo, Vol. VI, 1904/05, pag. 335.

Diés., On physiologically balanced Solutions. The Bulletin of the College of Agriculture, Tokyo, Vol. VII, 1906/08, pag. 395.

In diesen Abhandlungen finden sich auch weitere Literaturangaben.

10) Loew, O. u. Honda, S., Über den Einfluß wechselnder Mengen von Kalk und Magnesia auf $\backslash$ die Entwicklung der Nadelbäume. The Bulletin of the College of Agriculture, Tokyo, Vol. II, Nr. 6, 1896; zit. nach Botanisches Centralblatt 1898, LXXV, pag. 57.

11) Maki, S. and Tanaka, S., Regeneration of overlimed Soil. The Bulletin of the College of Agriculture, Tokyo, Vol. VII, 1906/08, pag. 61.

12) May, D. W., Experimental Study of the Relation of Lime and Magnesia to Plant Growth. U. S. Department of Agriculture. Bureau of Plant-Industry, Washington 1901, Bull. Nr. 1, pag. 37.

13) Namikawa, S., On the Lime Factor for Flax and Spinach. The Bulletin of the College of Agriculture, Tokyo, Vol. VII, 1906/08, pag., 57.

14) Sirker, G. N., Topdressing with Magnesium Sulphate. The Bulletin of the College of Agriculture, Tokyo, Vol. VII, 1906/08, pag. 613 . 
Takeuchi ${ }^{1}$ ) und Yokohama ${ }^{2}$ ) konnten die Entwicklung ihrer Versuchspflanzen günstig oder ungünstig beeinflussen, indem sie in dem Substrat, in dem alle Nährstoffe vorhanden waren, bloß das Verhältnis von $\mathrm{Ca}: \mathrm{Mg}$ variierten, während wir imstande waren, die Intensität der Erkrankung der Keimlinge von Phaseolus vulgaris in destilliertem Wasser zu steigern, indem wir Bohnen, welche Kalzium und Magnesium im Verhältnisse von 1:3 enthielten, nur Magnesiumnitrat aufnehmen ließen. Durch Zusatz von Kalziumnitrat zur Magnesiumnitratlösung ist man imstande, die Krankheitssymptome zu verändern, die Erkrankung zu verzögern und bei genügender Kalkzufuhr gänzlich zu verhindern.

Letzteres wurde erzielt, wenn die Keimlinge in einer Mischung von zwei Teilen einer $1 / 100$ Normallösung von $\mathrm{Ca}\left(\mathrm{NO}_{3}\right)_{2}$ und einem Teil einer gleichen Normallösung von $\mathrm{Mg}\left(\mathrm{NO}_{3}\right)_{2}$ kultiviert wurden.

Die Angaben in der Literatur über das günstigste $\frac{\mathrm{CaO}}{\mathrm{MgO}}$-Verhältnis schwanken zwischen 1-4, je nach der Art und dem Entwicklungszustand der Versuchspflanzen.

Bereits $\mathrm{B} o ̈ \mathrm{hm}^{3}$ ) war es gelungen, die giftige Wirkung von kohlensaurem Magnesium auf Phaseolus multiflorus durch Zusatz eines gleichen Teiles von kohlensaurem Kalk zu paralysieren.

In neuester Zeit ist es Meltzer und Auer ${ }^{4}$ ) gelungen, beim Kaninchen durch ein Magnesiumsalz hervorgerufene pathologische Erscheinungen durch ein Kalziumsalz aufzuheben.

$\mathrm{Loeb}^{5}$ ) benötigte bei seinen grundlegenden Versuchen mit befruchteten Eiern des Knochenfisches (Fundulus) zur Aufhebung der schädlichen Wirkung des Na-Ions Spuren zweiwertiger und noch geringere Mengen dreiwertiger Metallionen.

1) Takeuchi, T., Gypsum as a Manure. The Bulletin of the College of Agriculture, Tokyo, Vol. VII, 1906/08, pag. 583.

2) Yokohama, H., Why are poor sandy Soils often easily injured by Liming? The Bulletin of the College of Agriculture, Tokyo, Vol. VII, 1906/08, pag. 615 .

3) Böhm, J., l. c., Separatabdruck, pag. 10.

4) Meltzer, S. J. u. Auer, John, Die antagonistische Wirkung des Kalziums auf den Hemmungseinfluß des Magnesiums. Proceed. of the Royal Society 1908, ser. B., Vol. 80, pag. 260. Ref. Naturwissenschaftliche Rundschau, 1908, XXIII, Nr. 39 , pag. 498.

5) Loeb, Jacques, Über dem Einfluß der Wertigkeit und möglicherweise der elektrischen Ladung von Jonen auf ihre antitoxische Wirkung. Vorläufige Mitteilung. Archiv für die gesamte Physiologie 1901, Bd. 88, pag. 68. 
Osterhout ${ }^{1}$ ) konnte die giftige Wirkung von $\mathrm{MgCl}_{2}$ auf Spirogyra und Gemmen von Lunularia durch Zusatz von $\mathrm{KCl}$ aufheben. Kali allein wirkte giftig. Diese Entgiftung des zweiwertigen durch das einwertige Metall kam zustande bei Mischungen von $40 \mathrm{ccm}$ $3 / 8 \mathrm{M} . \mathrm{MgCl}_{2}, 100 \mathrm{ccm} 3 / 8 \mathrm{M} . \mathrm{KCl}$ und $20 \mathrm{ccm} \mathrm{H}_{2} \mathrm{O}$, resp. $40 \mathrm{~cm} \mathrm{MgCl}_{2}$ ${ }_{3 / 32}$ M., $100 \mathrm{ccm} \mathrm{KCl} \mathrm{3/32}$ M., und $20 \mathrm{ccm} \mathrm{H}_{2} \mathrm{O}$. Nach Loew und $\mathrm{Aso}^{2}$ ) kann die Giftigkeit der Magnesiumsalze durch Kalisalze verzögert aber nicht verhindert werden. Die Ursache dieser Verzögerung ist eine ganz andere, als bei der Verhinderung der Giftwirkung durch Kalziumsalze.

Benecke ${ }^{3}$ ) fand bei Versuchen mit Spirogyra, daß die Giftigkeit aller von ihm daraufhin untersuchten Kationen und Anionen durch Beigabe des Ions $\mathrm{Ca}$ aufgehoben oder doch vermindert werden kann.

Die schädliche Wirkung der Lösungen verschiedener Natronsalze und der Lösungen von Magnesiumsulfat und Magnesiumchlorid auf Zea mays, Andropogon sorghum, Avena sativa, Beta'vulgaris, Gossypium barbadense, Gossypium hirsutum, Lupinus albus und Triticum vulgare wurde von Kearny und Harter ${ }^{4}$ ) beschrieben. Die toxische Wirkung dieser Salze wurde durch Zusatz von Kalziumsulfat zu den Lösungen stark vermindert.

Wir und Daikuhara ${ }^{5}$ ) konnten bei Phaseolus die Giftwirkung von $\mathrm{Mg}$ durch die beiläufig doppelte Menge von Ca paralysieren. Die schädliche Wirkung des Magnesiums äußerte sich bei unseren Versuchen zuerst und am deutlichsten an den Organen, welche direkt mit der Lösung in Berührung kamen, also an den Wurzeln. Es ist daher trotz der entgegengesetzten Meinung $\mathrm{Bruchs}^{6}$ ) möglich, daß Magnesiumsalze auf Weizen und Buchweizen giftig wirken, denn obgleich sich die oberirdischen Teile seiner Versuchspflanzen in Magnesiumlösungen normal entwickelten, so sollen die Wurzeln doch geschädigt worden sein.

1) Osterhout, W. J. V., The, antitoxie Action of Potassium on Magnesium. University of California publications, Botany 1906, Vol. 2, Nr. 11, pag. 235.

2) Loew, O. u. A s.o, K., l. c., pag. 409.

Loew, O., 1. c., Flora 189\%, pag. 382, Über die Aufhebung der Giftwirkung von Magnesiumnitrat bei Spirogyra majuscula durch Calciumnitrat.

3) Benecke, W., l. c. Berichte der deutschen bot. Ges., pag. 331.

4) Kearny, T. H. and Hartér, L. L., l. c.

5) Daikuhara, G., l. c.

6) Bruch, Paul, Zur physiologischen Bedeutung des Kalziums in der Pflanze. Inaugural-Diss., Basel 1901. 
Berücksichtigt man alle diese Befunde und zieht man in Betracht,

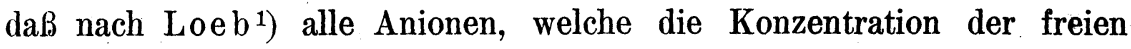
Kalziumionen vermindern, die Wirkung der giftigen Ionen wieder ermöglichen, so ist die Schlußfolgerung berechtigt, daß die Erkrankung der Pflanzen infolge von Kalkmangel auf äußerst komplizierten Vorgängen beruht.

Werden Pflanzen in kalkfreien Lösungen kultiviert, so wird, abgesehen von der direkten Schädigung der Wurzeln und der damit verbundenen Ernährungsstörung der Kalkmangel auch in den wachstumsfähigsten Teilen der Pflanzen die verschiedensten Veränderungen des Stoffwechsels zur Folge haben und direkte Wirkungen der dargebotenen Stoffe ermöglichen.

Die giftige Wirkung der freien organischen Säuren und der leicht löslichen Verbindungen derselben kann zur Geltung kommen ${ }^{2}$ ) und die Giftigkeit des Mg-Ions kann sich infolge des Auftretens größerer Quantitäten leicht dissoziierbarer Magnesiumverbindungen äußern.

Aber auch die anderen Ionen können nun einzeln in schädlichem Sinne, oder der schädlichen Wirkung anderer Ionen entgegenwirkend funktionieren.

$\mathrm{Zu}$ all diesen Kombinationen kommen noch andere, vielleicht auch noch unbekannte Veränderungen, welche in der Pflanze durch den Kalkentzug hervorgerufen werden $\left.{ }^{3}\right)$.

Das Resultat dieser gleichsinnigen oder entgegengesetzten Wirkungen tritt uns dann im Krankheitsbilde entgegen.

Diese Annahme, welche im großen und ganzen mit den Überlegungen Benekes ${ }^{4}$ ) und $\left(\mathrm{Czapeks}^{5}\right.$ ) übereinstimmt, soll die Basis für unsere weiteren Untersuchungen bilden.

1) Loeb, Jacques, Ist die erregende und hemmende Wirkung der Ionen eine Funktion ihrer elektrischen Ladung? Archiv für die ges. Physiologie 1902, Bd. 91 , pag. 248.

Ders., Über die antizytolytische Wirkung von Salzen mit zweiwertigen Metallen. Biochemische Zeitschrift 1907, Bd. V, 5. u. 6. H., pag. 351.

2) Loew, O., 1. c. Flora 1892.

Pfeffer, W., Pflanzenphysiologie I, 1897, pag. 427.

Schimper, A. F. W., Zur Frage der Assimilation der Mineralsalze durch die grüne Pflanze. Flora od. allgem. bot. Zeitung 1890, H. 3, pag. 207, 248.

3) Benecke, W., l. c., Berichte der deutschen bot. Ges. 1907.

Czapek, Fr., Biochemie der Pflanzen 1905, Bd. II, pag. 841, 850-852.

4) Benecke, W., 1. c., Berichte der deutschen bot. Ges. 1907.

5) Czapek, Fr., l. c. 
Vor allem wird zu untersuchen sein, in welcher Weise bei einer bestimmten Versuchspflanze in einem bestimmten Entwicklungsstadium die einzelnen Nährstoffe giftig oder entgiftend wirken. Denn es hat den Anschein, daß einerseits alle Komponenten der für die meisten assimilierenden Pflanzen notwendigen Verbindungen mit Ausnahme der Kalksalze, eine Giftwirkung und einige, wenn nicht alle eine entgegengesetzte, also entgiftende Wirkung hervorrufen können, ohne daß eine vollständige Aufhebung der Giftigkeit erreicht wird, während andererseits das Kalzium meist nur schwach giftige ${ }^{1}$ ), hingegen aber so stark entgiftende Eigenschaften zu besitzen scheint, daß die Toxizität jedes einzelnen oder der Summe der anderen Stoffe durch dasselbe paralysiert werden kann.

Es muß dann auch die Frage geprüft werden, ob nicht bei den Algen, welche nach Molisch ${ }^{2}$ ) des Kalkes zu ihrer Entwicklung entbehren können, anderen Nährstoffen diese entgiftende Wirkung zukoinmt.

Biologische Versuchsanstalt in Wien, Oktober 1908.

1) Während der. Drucklegung dieser Abhandlung ist eine neue Arbeit von Osterhout, W.J. V., „Die Schutzwirkung des Natriums für Pflanzen (Jahrb. f. wiss. Bot. 190́8, Bd. XLVI, H. 2, pag. 121), erschienen. Wir werden auf dieselbe bei einer anderen Gelegenheit zurückkommen.

2) Molisch, H., Die Ernährung der Algen. (Süßwasseralgen, I. Abhandlung.) Sitzungsberichte der Wiener Akademie. Math.-nat. Kl. 1895, Bd. CIV, I. Abt.

Ders., Die Ernährung der Algen. (Süßwasseralgen, II. Abhandlung.) Sitzungsberichte der Wiener Akademie. Math.-nat. Kl., 1896, Bd. CV, I. Abt. 